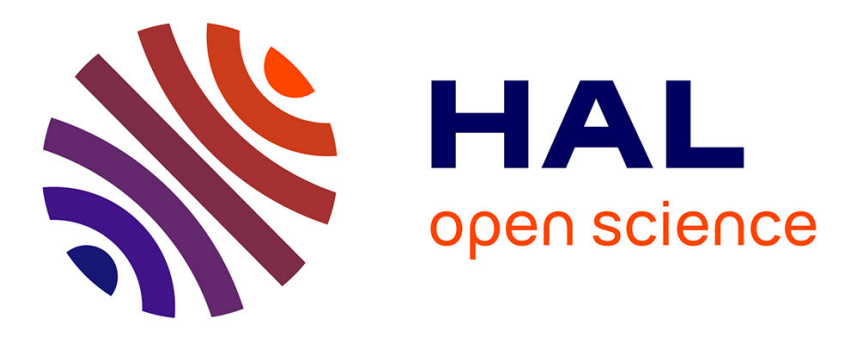

\title{
Le corps embryonnaire et fotal dans une approche relationnelle
}

\author{
Anne-Sophie Giraud
}

\section{To cite this version:}

Anne-Sophie Giraud. Le corps embryonnaire et fotal dans une approche relationnelle. Recherches familiales, 2014, 11, pp.9-17. 10.3917/rf.011.0009 . hal-01881014

\section{HAL Id: hal-01881014 \\ https://hal-univ-tlse2.archives-ouvertes.fr/hal-01881014}

Submitted on 25 Sep 2018

HAL is a multi-disciplinary open access archive for the deposit and dissemination of scientific research documents, whether they are published or not. The documents may come from teaching and research institutions in France or abroad, or from public or private research centers.
L'archive ouverte pluridisciplinaire HAL, est destinée au dépôt et à la diffusion de documents scientifiques de niveau recherche, publiés ou non, émanant des établissements d'enseignement et de recherche français ou étrangers, des laboratoires publics ou privés. 
Anne-Sophie Giraud

Doctorante sous la direction d'Irène Théry (UMR 8562, Centre Norbert Elias) et Dominique Memmi (UMR 7217, Cultures et Sociétés Urbaines)

EHESS de Marseille, Centre Norbert Elias (UMR 8562)

Proposition de texte pour la revue Recherches familiales, ${ }^{\circ} 11$, janvier 2014 : «Famille et corps : identité et transmission »

\section{Le corps embryonnaire et fotal dans une approche relationnelle.}

\section{Résumé}

L'anthropologie a longuement analysé la façon dont les techniques et notamment l'échographie et l'AMP (assistance médicale à la procréation), ont contribué à l'image de l'être prénatal comme « isolat», séparé en particulier du corps féminin. La personne, assimilée au corps embryonnaire, serait devenue une pure entité biologique isolée des relations sociales censées la conformer.

Cependant l'observation ethnographique des pratiques autour de l'AMP et de la mort périnatale nous révèlent que l'être prénatal et son corps, loin d'être de simples « donnés biologiques », sont toujours inscrits au sein d'un ensemble de relations sociales. Les façons de percevoir ce corps, de le décrire, de le qualifier et de le considérer, ne sont donc jamais indépendantes des liens actuels ou virtuels qui le relient avec les différentes personnes en rapport avec lui, à tel ou tel titre, selon tel ou tel statut.

\section{Introduction}

Le statut de l'être prénatal fait encore largement l'objet de débats au sein des sciences sociales et reste à ce jour non tranché tant dans les domaines éthique, philosophique ou encore juridique. C'est d'ailleurs l'une des questions les plus sensibles de l'actualité et l'une des plus controversées en droit contemporain, suscitant toujours de vastes polémiques. Ainsi, une multitude de propriétés, comme la conscience de soi, l'autonomie ou encore l'émergence de la ligne primitive sont autant de seuils utilisés pour dater et marquer entre autres le moment d'institution d'une personne. 
L'anthropologie a beaucoup analysé l'impact des techniques de visualisation, et tout particulièrement de l'échographie, sur l'image de l'être prénatal comme «isolat » séparé en particulier du corps féminin dans lequel il était autrefois enclos et enfoui ${ }^{1}$. L'AMP et notamment la FIV (fécondation in vitro) qui a pour conséquence de dissocier physiquement l'embryon de la femme, accentuent de manière radicale cette représentation de l'embryon « isolé ». Cependant l'observation ethnographique des pratiques d'AMP et de celles autour de la mort périnatale, révèle que l'être prénatal est toujours pris dans des réseaux relationnels et inscrit dans un monde institué. Relations d'une part à des professionnels, mais aussi et surtout en référence à la parenté, à l'ensemble des personnes impliquées dans la procréation, l'engendrement et/ou la filiation.

C'est ce que nous montrerons dans cet article grâce à une «approche relationnelle » inspirée de l'héritage maussien en matière d'analyse du genre et de la parenté ${ }^{2}$. Dans une première partie, après avoir montré le poids de l'idéologie des sociétés individualistes sur la manière de concevoir l'être prénatal et de manière plus générale la personne et le corps, nous exposerons l'intérêt de cette approche relationnelle. Elle permet de comprendre, objet de la seconde partie, comment l'être prénatal alterne entre diverses positions, entre enfant potentiel et pur matériau organique, selon le système de relations instituées dans lequel il se trouve inscrit. Nous mettrons en particulier l'accent sur le corps de l'être prénatal, un corps qui est à la fois le signe et l'instrument de la relation entre divers acteurs.

Nous nous sommes basée pour cela sur deux enquêtes. L'une est le fruit d'un travail de terrain débuté en France en 2010. Des observations et des entretiens semi-directifs ont été réalisés auprès de soignants et d'une vingtaine de personnes touchées par une mort périnatale. L'autre, réalisée en 2010 et 2011, est constituée d'entretiens semi-directifs auprès de soixante-dix professionnels de $1^{\prime} \mathrm{AMP}^{3}$.

Il s'agit ici d'un article présentant la construction à la fois théorique et empirique d'un objet d'étude, une étape indispensable et centrale pour toute analyse socio-anthropologique ${ }^{4}$.

\section{L'être prénatal, un être isolé ?}

\footnotetext{
1 Voir en particulier: Petchesky Rosalind P., «Fetal Images: The Power of Visual Culture in the Politics of Reproduction », in Feminist Studies, vol.13, n² (Summer), pp. 263-262, 1987.

2 Théry Irène, La distinction de sexe, Odile Jacob, 2007

3 Enquête collective réalisée dans deux grands centres d'AMP de Marseille par l'EHESS de Marseille, sous la direction d'Irène Théry. Elle portait sur le thème du genre en AMP et plus particulièrement sur les asymétries masculin/féminin. Avec une autre doctorante, Manon Vialle, nous avons constitué, sous la direction d'I. Théry, une petite équipe afin de transcrire intégralement les entretiens et de réaliser une exploitation secondaire des données.

${ }^{4}$ Mauss Marcel, Fauconnet Paul, « sociologie », Grande Encyclopédie, vol. 30, Société anonyme de la Grande Encyclopédie, Paris, 1901.
} 


\subsection{Le corps embryonnaire: un pur matériau biologique?}

Les débats sur le statut de l'embryon et du fœtus, qui ne vont pas sans remettre en cause la législation en vigueur, comme actuellement les discussions autour de la recherche sur l'embryon, interrogent la définition de la personne et par là de ce que nous entendons par le «corps ». A cette occasion, nous nous interrogeons tant sur le moment d'institution de la personne que sur le statut dont elle jouit par rapport aux individus préexistants.

Ce qui est particulièrement frappant dans l'ensemble de ces controverses, c'est que les définitions de la «personne embryonnaire » ou fœtale reposent sur une base biogénétique. Les discussions portent en effet principalement sur la détermination de seuils biologiques, de propriétés intrinsèques pouvant marquer le moment d'institution de la personne. Dans les débats autour de la recherche sur l'embryon notamment, c'est en effet sur ces seuils (émergence de la ligne primitive, implantation dans l'endomètre, etc.) que s'affrontent encore différentes idéologies comme en France ou en Grande-Bretagne lors de l'établissement de la Human and Fertilisation Embryology Act (1990).

Au début des années 1990, des anthropologues de la parenté observant ces débats, en particulier Marilyn Strathern ${ }^{5}$ et Sarah Franklin ${ }^{6}$, ont considéré qu'un tournant décisif s'était produit. Sous les effets notamment des progrès de la génétique, de l'AMP et de l'échographie, nous assisterions à des modifications radicales de la représentation de la personne et des rapports entre individus et parenté. Strathern et Franklin s'appuient pour cela sur le contraste entre les systèmes de valeurs et de représentations de l'individu dans les sociétés traditionnelles holistes et les sociétés modernes individualistes. Elles analysent ces changements notamment à travers l'étude de l'être prénatal. Ce dernier serait placé en dehors du faisceau de relations sociales qui caractérisait autrefois l'individu social inséré dans un système de parenté, un monde relationnel. Les relations se négocieraient désormais après coup, laissant place à la seule vérité biologique. S. Franklin ${ }^{7}$ va même jusqu'à affirmer que l'anthropologie classique n'aurait pas les outils nécessaires pour rendre compte de la situation contemporaine. Franklin et Strathern comparent donc la situation contemporaine non seulement au passé, centré uniquement sur le système de parenté, mais aussi aux sociétés « autres », dans lesquelles la personne serait éminemment relationnelle.

Malgré ses aspects très novateurs, différents reproches ont été adressés à cette perspective. Non seulement elle vient durcir un lieu commun en anthropologie, le clivage entre « l'Ouest et

\footnotetext{
5 Strathern Marilyn, After Nature. English kinship in the late twentieth century, Cambridge University Press, 1992.

6 Franklin Sarah, «Making Representations. The parliamentary debate on the Human Fertilisation and Embryology Act», in Technologies of Procreation. Kinship in the age of assisted conception, J. Edwards, S. Franklin, E. Hirsch, F. Price, M. Strathern, Routledge, London and New-York, 1999 (1993).

${ }^{7}$ Franklin Sarah, op. cit., 138, 1999 (1993).
} 
le reste », mais elle sépare aussi radicalement la modernité et la post-modernité de toutes les autres expériences socioculturelles. De plus, comme le soulignent de nombreux auteurs ${ }^{8}$ qui s'efforcent dès les années 1990 de replacer l'être prénatal dans un réseau relationnel, l'arrivée de ces technologies et notamment de l'AMP n'a eu pour conséquence ni de réduire le nombre d'acteurs autour de l'être prénatal ni de rendre les relations secondaires, voire inexistantes. Au contraire cela a eu pour effet d'en augmenter le nombre et de complexifier le réseau relationnel dans lequel il est inscrit ${ }^{9}$.

Enfin cette représentation de l'être prénatal « isolé » ne distingue pas clairement «l'idéologie individualiste » de la réalité sociale dans les sociétés modernes et repose sur une conception occidentale de la personne et du corps. Notre but ici n'est pas de déterminer le statut de l'embryon et du fœetus. Il est toutefois nécessaire de revenir sur cette définition de la personne, car l'approche de Strathern et Franklin mais aussi l'ensemble des débats autour de l'être prénatal reposent sur une définition présupposée et problématique de la personne et du corps, en fait une conception occidentale. Nous ne pouvons donc prétendre étudier l'être prénatal sans avoir au préalable éclairé ces conceptions de la personne.

En premier lieu, il est nécessaire de distinguer la personne au sens de «l'agent des actes humains » ou «agent empirique », qui désigne la réalité empirique et existe toujours et partout, de la personne au sens sociologique ou au sens du «sujet normatif des institutions ${ }^{10} »$, qui désigne une certaine idéalité normative, morale et juridique et varie selon les sociétés. Les conceptions du corps sont tout autant normatives que celles de la personne car elles dépendent de la grammaire sociale en usage ${ }^{11}$. A une personne au sens de l'idéalité normative correspond un corps spécifique. Dans les sociétés occidentales les discours produits sur la personne et le corps sont ceux d'un moi possesseur d'un corps, hérité en particulier du christianisme. La personne occidentale se pense comme agent ayant pour noyau une intériorité naturelle dont le corps est à la fois récipient et substance ${ }^{12}$. Dans les sociétés mélanésiennes au contraire, la personne est dite « relationnelle ». Elle est intégralement sociale car définie par des relations internes avec d'autres personnes. Le corps isolé par ses attributs intrinsèques, le corps «biologique » tel que nous le connaissons dans nos sociétés, n’y a aucun sens car le corps ne peut être séparé du tout cosmique dont il participe à travers sa place dans les relations ${ }^{13}$. Cette approche nous éclaire car la personne occidentale et son corps peuvent eux aussi être

\footnotetext{
${ }^{8}$ Dont: Porqueres i Gené Enric, «Individu et personne à la lumière des nouveaux contextes de parenté », in Défis contemporains de la parenté, Enric Porqueres i Gené (sous la direction), éd. de l'Ehess, 2009.

9 Bateman-Novaes Simone, Salem Tania, «Embedding the Embryo », in The Future of Human Reproduction: Ethics, Choice and Regulation, J. Harris, S. Holm (eds), Oxford University Press, pp. 101-126, 1998.

${ }^{10}$ Dumont Louis, Homo hierarchicus. Essai sur le système des castes, Paris Gallimard, 1967.

11 Théry Irène, op. cit., pp. 415-416, 2007.

12 Breton Stéphane, Coquet Michèle, Houseman Michael, Schaeffer Jean-Marie, Taylor Anne-Christine, Viveiros de Castro Eduardo, Qu'est-ce qu'un corps?, Ed. Flammarion, Musée du Quai Branly, 2006

13 Strathern Marilyn, The Gender of The Gift, Berkeley-Londres, University of California Press, 1988.
} 
considérés comme relationnels. Le corps occidental est en effet à la fois le signe et l'instrument de la relation entre le sujet de l'individualisme occidental - ce moi possesseur d'un corps - et Dieu, ou tout autre principe génératif (comme le code génétique) qui s'y serait incarné. Le corps humain, la personne et l'engendrement ne sont donc pas des entités ou des actes purement biologiques mais sont toujours déjà investis de sens, inscrits dans un système de relations instituées et notamment au sein d'un système de parenté qui les précède logiquement. Le corps doit par conséquent être étudié comme la matérialisation, le signe et l'instrument, d'un système relationnel.

\subsection{Approche « relationnelle maussienne ».}

C'est grâce à une approche relationnelle inspirée de l'héritage maussien que nous allons mettre à distance cette perception occidentale de la personne et du corps, une étape indispensable à l'étude de l'être prénatal.

La particularité d'une telle approche est bien sûr la prise en compte des réseaux sociaux mais aussi et surtout la place donnée à l'institution, c'est à dire aux systèmes d'institutions auxquels se réfèrent les individus. Une telle approche appréhende donc toujours la réalité sociale sur deux niveaux : ce que font les acteurs et le système implicite auquel ils font référence et qui peut être soumis à de fortes turbulences. Une société d'après M. Mauss ${ }^{14}$, est en effet un «tissu concret de relations » dont la plupart sont à un certain degré instituées, inscrites dans la temporalité, référées à des valeurs et des significations communes permettant aux individus d'agir en référence à des règles - qu'ils peuvent d'ailleurs ne pas respecter ou ne connaître que partiellement. Il est donc toujours artificiel de séparer l'individu du « tout » concret qu'est la société à laquelle il participe comme personne, c'est-à-dire comme agent des actes humains. Pour résumer, les statuts modalisent des manières d'agir ; ils sont « relationnels », dans le sens où ils ne peuvent exister indépendamment de la relation aux autres, et ne sont pas des attributs intrinsèques des individus, même acquis ${ }^{15}$.

Loin d'appréhender l'être prénatal comme un être «isolé » en ne prenant en compte que ses attributs intrinsèques, ses propriétés ou ses caractères internes, nous étudierons donc son statut « relationnel », c'est à dire les relations que peuvent entretenir avec lui d'autres personnes possédant elles-mêmes un statut. Mais s'intéresser à son statut relationnel ne signifie pas pour autant oublier le corps, mais que le corps lui-même peut être abordé comme « un ensemble de relations ${ }^{16} »$, nous l'avons vu.

\footnotetext{
${ }^{14}$ Mauss Marcel, Essai sur le don, ed. PUF, 2007 (1 ${ }^{\text {ère }}$ ed. 1924-1925).

15 Théry Irène, 2007, op. cit.

${ }^{16}$ Breton et al., 2006, op. cit.
} 
Ces relations sont variables mais sont elles-mêmes référées à des normes et des règles communes, en particulier à l'ensemble des dispositifs juridiques qui peuvent changer et être contradictoires. La manière dont la loi définit l'être prénatal module en effet la relation qu'on entretient avec lui, tout en prenant en compte que le monde social évolue et produit des statuts. De même, ces relations ne dépendent pas principalement des propriétés intrinsèques de l'être prénatal puisque des qualités très différentes peuvent être données à un même substrat. Elles dépendent avant tout du sens que nous donnons à cette relation, une relation elle-même inscrite dans un contexte social, culturel et historique. Ceci est très bien illustré par L. Boltanski ${ }^{17}$, dans sa description d'une séance d'échographie. Selon qu'elle est réalisée en vue d'un avortement ou dans le contexte d'une grossesse "normale", l'échographie est utilisée par le soignant pour transformer quelques cellules en un bébé ou en rien. Ainsi, toutes les actions de ces personnes, soignants, géniteurs et/ou parents (la manière de nommer, de se comporter avec ou encore de se représenter) et les qualités qu'ils attribuent à l'être prénatal, vont tendre à l'inscrire dans la parenté, la vie sociale et plus largement l'humanité, ou à l'inverse à ne pas l'inscrire. Toutefois le corps influence aussi la relation que nous pouvons entretenir avec cet être, car les parents et les soignants possèdent une palette de choix beaucoup plus grande au début de la conception qu'à la fin, avec le fœus près de naître.

Les débats sociopolitiques sont structurés par des idéologies pour lesquelles l'engendrement n'est pas un processus mais un état de fait. Certaines ne font pas en effet de distinctions entre les quelques cellules que forment un embryon et le fotus près de naître. Elles n'intègrent pas cette dimension du développement progressif de l'être prénatal car elles n'ont aucun sens pour elles. Pour l'Eglise catholique par exemple, l'être humain en gestation est déjà un enfant et ce dès la conception. Nous affirmons au contraire que prendre en compte le corps et son développement progressif est essentiel dans l'étude de l'être prénatal. C'est pour cette raison que nous avons choisi de l'étudier aux deux pôles extrêmes du processus de gestation. Cela permet notamment d'éviter le biais constant d'assimiler tel ou tel moment dans le processus d'institution progressive de l'humanité comme celui renvoyant l'être prénatal soit du côté des choses soit du côté des personnes. D'ailleurs, bien qu'il y ait des différences capitales entre un embryon et un fœetus près de naître, les acteurs sont confrontés dans ces extrêmes à des mécanismes et dilemmes similaires. D'autant plus que nous avons affaire dans les deux cas, avec l'embryon in vitro et le mort-né, à des êtres en « marge ».

A partir d'enquêtes que nous avons réalisées, nous allons ainsi montrer comment à ces deux moments du processus de gestation, à la conception avec l'embryon en AMP et à la fin avec le fœtus mort-né, le corps de l'être prénatal est modelé par les actions d'acteurs qui

\footnotetext{
${ }^{17}$ Boltanski Luc, La condition foetale. Une sociologie de l'engendrement et de l'avortement, ed. Gallimard, 2004, pp. 176-177.
} 
l'inscrivent dans un statut relationnel, des actions elles-mêmes modelées par le corps de l'être prénatal et inscrites dans un monde institué.

\section{Corps embryonnaire, corps fotal: agir sur le corps et construire une relation.}

Nos observations ethnographiques des pratiques autour de l'AMP et de la mort périnatale nous ont révélé que l'être prénatal est toujours pris dans des réseaux relationnels. Relations d'une part à des professionnels qui ont à un certain moment, du fait de leur statut, le pouvoir d'observer, de sélectionner, de détruire ou d'humaniser cet être. D'autre part et surtout, en référence à la parenté, relations à l'ensemble des personnes impliquées dans la procréation, l'engendrement et/ou la filiation et qui de ce fait, ont elles aussi tout un ensemble de pouvoirs et de devoirs à l'égard de cet être.

Nous présentons ici les données d'une enquête en cours. Il s'agira, objet d'un futur travail, de l'étendre aux patients en AMP et donc d'étudier plus spécifiquement ceux qui placent (ou non) l'être prénatal dans le système de parenté.

\subsection{L'embryon dans le processus d'AMP.}

Lors de notre enquête auprès des professionnels de l'AMP, nous nous sommes aperçus que soignants et patients sont en permanence confrontés à l'existence de l'embryon, rendu plus accessible par les techniques médicales de procréation. Le sens qu'ils donnent à cette relation, les qualités qu'ils attribuent à cet être, modulent leur manière de percevoir l'embryon et le corps embryonnaire. Dans le même temps, les caractéristiques internes de l'embryon ainsi que son état, modifient les perceptions qu'en ont les individus. Selon qu'il est inscrit ou désinscrit d'un projet parental, congelé, in vitro, in utero, ou encore porteur d'une maladie génétique, une même personne va être amenée à voir cet embryon de manière différente. Les individus n'ont donc en général pas de représentations fixes de l'embryon dictées par des critères sociaux définis. Elles dépendent avant tout du contexte relationnel dans lequel ils se trouvent inscrits ainsi que du corps de l'embryon et de son état.

Etudions le cas précis de la sélection et de la congélation des embryons in vitro et de leurs effets à la fois sur les soignants et sur les patients.

Avant le transfert des embryons in vitro et avant que certains ne soient congelés, les biologistes et les techniciens de laboratoire opèrent une sélection entre les embryons 
susceptibles de se développer et les autres. Les embryons sont sélectionnés en fonction de «critères de beauté », en fait des critères de développement cellulaire. Seuls ceux qualifiés de « beaux », susceptibles de se développer, sont transférés ou congelés, les autres sont détruits. L'enquête a donc permis de mettre en lumière deux aspects qui ne font même pas l'objet de débat, mais qui sont pourtant capitaux : la sélection et la congélation changent radicalement le statut des embryons in vitro.

La sélection et les critères visuels sur lesquels elle repose, construisent les normes de traitements de l'embryon. Dès lors que les embryons sont sélectionnés, ils sont investis d'un sens différent et traités de manière différente. Le «bel » embryon est traité comme un être de qualité, destiné à devenir potentiellement un enfant. Il bénéficie de toutes les attentions des soignants et des patients. L'embryon de mauvaise qualité est en revanche considéré comme un pur matériau organique, un déchet qui est éliminé sans même qu'il soit nécessaire d'en informer le couple.

Il en est de même pour la congélation. Si jeter les embryons les moins «beaux» est couramment accepté avant la congélation et reste à la seule discrétion des soignants, après la phase de congélation, l'accord formel des deux membres du couple ${ }^{18}$ est impératif pour détruire le moindre embryon.

La congélation change aussi pour les patients la manière de percevoir l'embryon et la relation que l'on entretient avec lui, en particulier lorsque les termes de la relation couple/embryons ont été modifiés. En général, les couples se représentent leurs embryons comme des «enfants potentiels ». Ceci est particulièrement vrai lorsque ceux qui possèdent des embryons congelés sont déjà devenus parents après l'implantation d'autres embryons. Ils sont devenus des «parents » et le ou les embryons implantés, des enfants. Les embryons congelés restants deviennent alors des frères et sœurs de ces enfants. Le système relationnel dans lequel ils sont inscrits ayant été totalement modifié, les embryons ont de fait acquis une certaine matérialité. De cellules pouvant potentiellement devenir un enfant, ils sont devenus des enfants ou des enfants potentiels.

Il est important de noter que ces mêmes couples se questionnent également sur l'âge réel de ces enfants à naître par rapport aux enfants déjà nés. Conçus le même jour mais nés à des dates différentes, ils seraient pour eux, comme nous le rapporte une gynécologue, des «faux jumeaux à temps distant». La congélation, en modifiant l'espace-temps, modifie là encore la relation entre les embryons congelés et les enfants déjà nés. La FIV et la congélation ont pour conséquences, que l'âge de l'embryon n'est pas l'âge de l'embryon activé comme fœus potentiel dans le cadre de la grossesse. Ce qui a été défait, c'est la simultanéité entre la conception de l'embryon et le début de la grossesse, condition pour que cet embryon se

\footnotetext{
${ }^{18}$ Ou accord du survivant en cas de décès.
} 
développe comme futur fœtus. Ce qui est présenté comme un problème de propriétés intrinsèques de l'embryon (quel âge a-t-il ?) repose en fait sur un changement relationnel, celui de l'embryon au corps gestationnel.

Tout au long d'un parcours d'AMP, le corps de l'embryon est donc modifié : in vitro, in utero, congelé, surnuméraire, inscrit ou non dans un projet. Ces états ainsi que les actions des différents acteurs, vont modifier les relations autour de cet embryon et les représentations qu'en ont les couples, oscillant entre enfant potentiel et pur matériau organique, inscrit ou non dans une parenté.

\subsection{Le fotus mort-né.}

Depuis le début des années 1990, le statut du fotus mort in utero n'a cessé de se métamorphoser. Son changement de statut a bien sûr été opéré sur le plan juridique, mais il apparaît avant tout au niveau «corporel », en particulier par le soin apporté au cadavre du mort-né. Ces transformations ont affecté en particulier le fœtus des états « intermédiaires », à savoir celui situé entre la limite légale de l'avortement ${ }^{19}$ et 22 SA (semaines d'aménorrhée) ${ }^{20}$, appelé « enfant sans vie » né vivant ou mort mais non viable, ainsi que le fœtus mort-né mais qui aurait été viable ${ }^{21}$.

Auparavant le corps du fœetus mort in utero était considéré comme un déchet hospitalier qui n'appartenait à personne et qui ne pouvait faire l'objet d'un rituel funéraire ${ }^{22}$. Il était alors impensable de le montrer à la mère. Il était destiné à la crémation avec les autres déchets hospitaliers et ne pouvait être que « déclaré sans vie ». Cependant depuis 1993, ce fotus n'était plus assimilé à un déchet anatomique. Il bénéficiait d'une crémation non plus dans un incinérateur hospitalier, mais dans un crématorium selon une procédure codifiée. Il était - et il est toujours - enregistré uniquement dans la partie " décès " des registres d'état civil, l'inscription étant laissée à la discrétion des parents. Il était en revanche exclu de le considérer comme une personne décédée, faute de naissance. Toutes ces pratiques allaient dans le sens d'une réification du fœetus mort in utero.

Aujourd'hui ces pratiques ont été complètement bouleversées. En effet, grâce aux deux décrets du 20 août $2008^{23}$ publiés après un revirement de la Cour de cassation le 6 février 2008 et à la

\footnotetext{
${ }^{19}$ Fixée à 14 SA depuis 2001.

${ }^{20}$ Seuil de viabilité défini par l'Organisation Mondiale de la Santé (OMS) en 1977.

${ }^{21}$ F. Michaud-Nérard, La révolution de la mort, Paris Vuibert, pp.40-43, 2007.

${ }^{22}$ Ce traitement concernant uniquement les fœtus mort in utero de moins de 28 SA, ou 180 jours de gestation depuis 1993. Ce seuil a ensuite été abaissé à 22 SA par la circulaire n²001/576 du 30 novembre 2001 et l'arrêté du 19 juillet 2002. Juridiquement, en dessous de ces seuils, le fœtus mort in utero n'avait aucune réelle existence juridique.

23 JO du 22 août 2008.
} 
circulaire du 19 juin 2009, il est désormais possible, si les couples le désirent, de délivrer un acte «d'enfant sans vie » pour les fotus morts de moins de $22 \mathrm{SA}^{24}$, et éventuellement de les inscrire dans le livret de famille.

Dans un contexte où le statut de ces «enfants sans vie » est désormais laissé à la convenance des couples qui choisissent ou non de l'inscrire dans la parenté et donc dans l'humanité, la manière de voir cet être dépend alors du sens que donnent les acteurs à cette relation. Cet « enfant sans vie » peut soit être considéré comme le produit d'une fausse-couche, un être que l'on souhaite oublier, soit au contraire comme un enfant que l'on reconnait, que l'on inscrit dans sa famille, à défaut d'une filiation juridiquement reconnue ${ }^{25}$. Dans ce dernier cas, la relation entre le couple et le mort-né se construit en partie grâce à son corps qui devient alors le signe et l'instrument de la relation entre l'« enfant» et ses «parents », mais aussi entre le couple, les soignants et la société. Ces relations ne dépendent pas principalement de propriétés intrinsèques du fœetus mort-né, nous l'avons vu, mais dépendent avant tout du sens que nous donnons à cette relation. Toutefois ces propriétés corporelles, inscrites dans un monde institué, influencent aussi la relation que l'on peut entretenir avec l'être prénatal. Nous avons ainsi pu observer que généralement la relation avec le fœtus mort-né ne pouvait pleinement se réaliser que lorsqu'un corps sexué et entier est disponible et présentable, deux conditions également requises pour la délivrance d'un certificat d'accouchement et donc d'un acte «d'enfant sans vie ».

Une telle perspective opère un décentrement qui nous conduit à porter notre attention non plus seulement sur le seul fœtus, son statut juridique et ontologique, mais aussi sur l'ensemble des relations qui le lie à ceux et celles qui participent à cette transformation d'un simple fœus à un «bébé ».

Tout l'enjeu des soignants comme l'a montré D. Memmi ${ }^{26}$, est alors de construire cet être comme une «personne ", c'est à dire comme un être en relation avec d'autres personnes et notamment ses géniteurs. Ils vont agir conjointement sur le corps du fœtus mort afin de construire cette relation et l'inscrire ou non dans la parenté. Cette construction se fait bien sûr par le biais de la dation d'un prénom, de l'inscription de l'enfant né sans vie sur le registre des décès ainsi que sur le livret de famille. Elle se fait également par le biais de la présentation du mort-né aux «parents », que cette présentation se fasse directement ou indirectement.

Avant d'être présenté aux parents, le mort-né est tout d'abord emmené par les soignants hors de la salle d'accouchement afin d'être lavé et vêtu. Une attention toute particulière est apportée

\footnotetext{
24 Sous condition toutefois de la délivrance d'un certificat médical d'accouchement, impliquant le recueil d'un corps formé et sexué. Ainsi les situations d'interruption volontaire de grossesse, de fausse-couche spontanée précoce survenant en deçà de la quinzième semaine d'aménorrhée ne répondent pas en principe à ces conditions.

${ }^{25}$ Seule la naissance d'un enfant vivant et viable donne accès à la personnalité juridique et donc à la qualité de personne. L'acte d' « enfant sans vie » n'ouvre pas droit à la filiation et par conséquent au nom de famille.

${ }^{26}$ Memmi Dominique, La seconde vie des bébés morts, éditions de l'Ehess, 2011.
} 
aux vêtements, symboles importants de l'humanité et de la personne, et donc de l'inscription dans un tissu relationnel. Ainsi les soignants encouragent les parents à en apporter, en particulier dans le cas d'une IMG (Interruption médicale de grossesse) où l'intervention est programmée et permet de s'y préparer. L'hôpital peut également en fournir car non seulement les vêtements permettent de donner un visage plus « humain » au fœtus mais ils permettent aussi de cacher certaines malformations trop visibles. Lorsque l'enfant est vraiment trop petit et qu'on n'a pas réussi à lui trouver des vêtements à sa taille, il est alors soigneusement enveloppé dans un drap ou une couverture. Si la présentation de l'enfant aux parents n'est pas possible pour diverses raisons - le corps présentant par exemple une malformation jugée trop choquante - un langage métonymique est alors utilisé. On en présente seulement une partie comme des mèches de cheveux ou des empreintes de pieds et/ou de mains, toute chose qui se rattache à «l'enfant».

Le mort-né est ensuite amené aux parents, porté dans les bras d'un soignant comme un enfant « normal », ou lorsqu'il est trop petit ou en trop mauvais état, présenté sur une table. Il s'agit donc d'une présentation humanisée et « humanisante » d'un fœtus qui devient un enfant quand il est prénommé, nettoyé et habillé. Par ces actes, en instituant le mort-né comme une personne, les soignants instituent donc par la même occasion le couple comme ses parents. C'est un processus qui trouve son aboutissement lorsque le couple désire organiser des obsèques et procéder à l'inhumation. En le traitant comme une personne décédée et non comme un déchet hospitalier, le couple inscrit alors cet être comme leur enfant aux yeux de la société.

\section{Conclusion}

L'embryon oscille donc sans cesse du statut de «cellules » à celui « d'enfant potentiel » et le mort-né, du statut de simple produit de fausse-couche à celui de bébé à part entière. La manière de se représenter l'être prénatal dépend en fait essentiellement des situations auxquelles les acteurs doivent faire face.

L'embryon en AMP et le fœus mort révèlent en outre que le corps sert d'instrument pour établir une relation entre «enfant» et «parent», entre couple et soignants, tout en étant le signe de cette relation. Ainsi, le corps embryonnaire ou fœtal peut être appréhendé comme un ensemble de relations. Loin d'être un pur matériau organique que le culturel viendrait lire et interpréter, le corps est donc toujours déjà investi de sens et en reste symboliquement marqué.

Etudier l'être prénatal à la fois selon sa place dans tel ou tel système de relations et le contexte socio-historique dans lequel il se trouve (contexte juridique, social, etc.) permet donc de mieux 
décrire et analyser non seulement tout le spectre de la palette d'attitudes observées chez les patients et les soignants, mais aussi de mieux comprendre les représentations, elles-mêmes évolutives, que chacun se fait de l'être prénatal.

Ce travail a pu être réalisé grâce à l'assistance fournie par l'association AECF et le fonds de recherche du laboratoire Ipsen. 